\title{
Aptamer-Conjugated Gold Nanoparticles Targeting Epidermal Growth Factor Receptor Variant III for the Treatment of Glioblastoma
}

This article was published in the following Dove Press journal: International Journal of Nanomedicine

\author{
Li Peng, 1,2,* Yanling Liang, ', \\ Xinxin Zhong,' Zhiman Liang, ,2 \\ Yinghong Tian, ${ }^{3}$ Shuji Li,' \\ Jingxue Liang, ${ }^{4}$ Ransheng Wang, ${ }^{4}$ \\ Yuqi Zhong, ${ }^{4}$ Yusheng Shi, ${ }^{5}$ \\ Xingmei Zhang (iD) \\ 'Key Laboratory of Mental Health of the Ministry \\ of Education, Guangdong-Hong Kong-Macao \\ Greater Bay Area Center for Brain Science and \\ Brain-Inspired Intelligence, Guangdong Province \\ Key Laboratory of Psychiatric Disorders, \\ Department of Neurobiology, School of Basic \\ Medical Sciences, Southern Medical University, \\ Guangzhou 510515, People's Republic of China; \\ ${ }^{2}$ The Fifth Affiliated Hospital, Southern Medical \\ University, Guangzhou 510900, People's Republic \\ of China; ${ }^{3}$ Experiment Teaching \& Administration \\ Center, School of Basic Medical Sciences, \\ Southern Medical University, Guangzhou 510515, \\ People's Republic of China; ${ }^{4}$ The First Affiliated \\ Hospital, Southern Medical University, \\ Guangzhou 5 105I5, People's Republic of China; \\ ${ }^{5}$ Department of Radiation Oncology, Nanfang \\ Hospital, Southern Medical University, \\ Guangzhou 510515, People's Republic of China \\ *These authors contributed equally to this work
}

Correspondence: Xingmei Zhang Key Laboratory of Mental Health of the Ministry of Education, Guangdong-Hong Kong-Macao Greater Bay Area Center for Brain Science and Brain-Inspired Intelligence, Guangdong Province Key Laboratory of

Psychiatric Disorders, Department of Neurobiology, School of Basic Medical Sciences, Southern Medical University, No. 1838 Guangzhou Avenue, Guangzhou 5105I5, People's Republic of China Email zxmray@hotmail.com

Yusheng Shi

Department of Radiation Oncology, Nanfang Hospital, Southern Medical University, No. 1838 Guangzhou Avenue, Guangzhou 510515, People's Republic of China

Email syszxm@hotmail.com
Purpose: In this study, we constructed novel brain-targeting complexes (U2-AuNP) by conjugating aptamer U2 to the gold nanoparticle (AuNPs) surface as a promising option for GBM therapy.

Materials and Methods: The properties of the U2-AuNP complexes were thoroughly characterized. Then, we detected the in vitro effects of U2-AuNP in U87-EGFRvIII cell lines and the in vivo antitumor effects of U2-AuNP in GBM-bearing mice. Furthermore, we explored the inhibition mechanism of U2-AuNP in U87-EGFRvIII cell lines.

Results: We found that U2-AuNP inhibits the proliferation and invasion of U87-EGFRvIII cell lines and prolongs the survival time of GBM-bearing mice. We found that U2-AuNP can inhibit the EGFR-related pathway and prevent DNA damage repair in GBM cells.

Conclusion: These results reveal the promising potential of U2-AuNP as a drug candidate for targeted therapy in GBM.

Keywords: SELEX, EGFRvIII, GBM, therapy

\section{Introduction}

Glioblastoma (GBM), also known as high-grade glioma, is an aggressive and lethal primary brain cancer with clinical survival rarely longer than 14 months when treated with the standard therapy of tumor resection followed by temozolomide as well as radiotherapy. ${ }^{1,2} \mathrm{GBM}$ is characterized by excessive proliferation, invasiveness, and resistance to radiation therapy or chemotherapy. Alteration in core signaling pathways in GBM genetic profiling, such as RTK/RAS/PI3K/PTEN, P53/ARF/MDM2, and $\mathrm{RB} / \mathrm{DKN} 2 \mathrm{~A}$ pathways, drives the existence of GBM heterogeneity, which causes the recurrence of GBM. ${ }^{3-5}$ Among these pathways, epidermal growth factor receptor (EGFR) is one of the most critical nodes; EGFR variant III (EGFRvIII, EGFR type III, or $\triangle \mathrm{EGFR}$ ) is the most commonly overexpressed EGFR family member in GBM, which causes tumor proliferation. ${ }^{4,6}$ Previous studies have demonstrated that the expression of EGFRvIII is closely correlated with the poor prognosis and resistance of therapy. Many studies and our previous work have shown that EGFRvIII is selectively expressed in tumor cells but not in normal cells, which indicates that EGFRvIII is a suitable molecular target for GBM therapy. ${ }^{7-9}$

Aptamers, single-stranded oligonucleotides, can be selected from a large random single-stranded DNA (ssDNA) or RNA library by systematic evolution of ligands by exponential enrichment (SELEX). Aptamers are known as chemical antibodies, which 
are characterized by low molecular weight, immunogenicity, and toxicity and high affinity and specificity. ${ }^{10,11}$ To effectively target EGFRvIII, we acquired a DNA aptamer named $\mathrm{U} 2$ in our previous work, which confirmed that the aptamer U2 can inhibit proliferation, migration and invasion and downregulate EGFR-related signaling pathways in GBM. ${ }^{9,12}$

The blood-brain barrier (BBB), a cell-based membrane, is covered by a sheath of vascular endothelial cells and perivascular astrocyte end-feet connected through tight junctions. ${ }^{13,14}$ According to previous research on pharmacokinetics, the BBB limits most chemotherapeutic and biological agents in peripheral blood from passing through and penetrating tumors in the brain. The existence of BBB hinders free access to most molecules, which leads to a high attrition rate of therapeutic drugs in the central nervous system (CNS). However, BBB pathology appears to have altered permeability when tumors occur. ${ }^{15,16}$ Taking advantage of the change in the BBB enables the design of a system with effective delivery into the cerebrum for targeted therapy of GBM.

Gold nanoparticles (AuNPs) are one of the most common noble metal inorganic nanoparticles and are characterized by controllable particle diameters, large surface/volume ratios, ease of modification, and great potential as new generation nanomedicine materials for integrated diagnosis and therapy of disease. ${ }^{17,18}$ It has been reported that appropriately sized gold nanoparticles can cross the BBB, providing a new strategy for intracranial targeted drug delivery therapy. It is unfortunate that gold nanoparticles lack targeting ability and that aptamers show weak transmembrane penetration. ${ }^{19}$ To promote more efficient crossing of aptamer U2 across the BBB to reach GBM in the cerebrum, we conjugated U2 with AuNPs through a gold-sulfur covalent bond. In the current study, we investigated whether U2-AuNP complexes might be a new type of drug candidate for GBM therapy by detecting proliferation and invasion in vitro and the antitumor effects in vivo in the GBM model. Furthermore, we preliminarily explored the activation of relevant signaling pathways to obtain another in-depth insight into U2-AuNP complexes in GBM. Our findings show the desired results in GBM treatments in vivo and in vitro, suggesting that U2-AuNP complexes are a new type of drug candidate for GBM therapy.

\section{Materials and Methods}

\section{Materials}

Common reagents were purchased from Sigma-Aldrich or ThermoFisher Scientific. NAP-5 columns were obtained from GE Healthcare (Illustra, Buckinghamshire, UK). Gold nanoparticles are purchased from BBI Group (lot: EM. GC15-100ML) (Crumlin, United Kingdom). Aptamers U2 were selected in the GN library containing a central random region of 40-nt via the Cell-SELEX process. ${ }^{20}$ Aptamer Scr1, a random nonsense DNA oligonucleotide, was the negative control. The sequence of $\mathrm{U} 2$ is: ATCCAGAGTGACGC AGCATTTTGACGCT TTATCCTTTTCTTATGGCGGGA TAGTTTCGTGGACACGGTGGCTTAGT; the sequence of the aptamer Scrl is: GCCAGCAACGGGCTTAGTTTC GTTATTTATACTTCGTCGCTTGAC GGAACGCGTGTT GGGCGTATTATTTTGCAAG.

\section{Synthesis of the Aptamer-AuNP Complex}

First, a 5' thiol-modified aptamer U2 (ThermoFisher Scientific) was dissolved in PB buffer and deprotected with TCEP in the dark. After $60 \mathrm{~min}$, the solution was transferred to a NAP-5 column to separate the aptamer and TCEP, thereby the aptamer U2 was purified. A large quantity of gold nanoparticles (AuNP), $4.38 \times 10^{-9} \mathrm{M}$, was added to 1.60 $\mathrm{x} 10^{-6} \mathrm{M}$ deprotected aptamer and reacted for $20 \mathrm{~min}$ at room temperature. Then, 0.1 M PB buffer containing 1\% SDS was added to allow continuous reaction. $\mathrm{NaCl}(2 \mathrm{M})$ was added into the sample at the appropriate volume step by step until the final concentration of $\mathrm{NaCl}$ was adjusted from $0.1 \mathrm{M}$ to $0.7 \mathrm{M}$ in seven steps, with one step every $20 \mathrm{~min}$. When the sample was reacted overnight at room temperature, the mixture was centrifuged for $30 \mathrm{~min}$ at $14,500 \mathrm{~g}$ three times to remove the free aptamer or AuNP. After the washing steps, the resulting apt-AuNPs were resuspended in $0.01 \mathrm{M} \mathrm{PB}$ containing $0.1 \mathrm{M} \mathrm{NaCl}$.

\section{Characterization of apt-AuNP Conjugates}

The particle size was detected using Malvern dynamic light scattering. Ultraviolet (UV)-visible spectroscopy was recorded on a UV-visible NanoDrop spectrometer (ThermoFisher Scientific). Transmission electron microscopy (TEM) and an $0.8 \%$ agarose gel were used to qualitatively detect whether aptamer U2 was conjugated onto the surface of AuNPs.

\section{Cell Proliferation Assay}

U87-EGFRvIII cell line (kindly provided by Dr. Webster Cavenee, Ludwig Cancer Institute, San Diego, CA) was grown in DMEM supplement with $10 \%$ FBS. The use of the cell line had ethical and institutional review board approval. Cell proliferation was analyzed using the Cell Light EdU DNA Imaging Kit (Guangzhou RiboBio, China). U87-EGFRvIII cells were seeded at $10^{4}$ per well 
with a 96-well plate and incubated with five replicates for each condition. After overnight incubation at $37^{\circ} \mathrm{C}$, the cells treated with U2-AuNP or AuNPs were incubated for another $24 \mathrm{hr}$. Then, 5-ethynyl-2'-deoxyuridine (EdU) $(50 \mu \mathrm{M})$ was added to the 96-well plate, and the cells were cultured for an additional $2 \mathrm{hr}$. Then, the cells were fixed with 4\% paraformaldehyde, washed with glycine $(2 \mathrm{mg} / \mathrm{mL})$, and permeabilized with $0.5 \%$ Triton X-100 for 10 min. Next, the cells were washed with PBS buffer three times and stained with fresh Apollo stain in the dark for $30 \mathrm{~min}$. Finally, the cells were incubated with Hoechst 33342 stain in the dark for 20 min at $37^{\circ} \mathrm{C}$ to visualize the nucleus using a laser scanning confocal microscope. The proliferation rate of cells was calculated as EdU-positive cells/Hoechst-stained cells x 100\%.

\section{Cell Invasion Assay}

U87-EGFRvIII cells were planted in 24-well Transwell plates (Corning Incorporated, Corning, NY) precoated with Matrigel (BD Biosciences, NJ) overnight. Cells were treated with U2-AuNP or AuNPs for another $24 \mathrm{hr}$ and then washed with PBS buffer three times and stained with crystal violet for $2 \mathrm{hr}$. Images were taken and analyzed using an inverted fluorescence microscope and ImageJ software (NIH).

\section{Western Blot Analysis}

The Western blot analysis was performed as previously described. U87-EGFRvIII cells were cultured in DMEM supplement with $10 \% \mathrm{FBS}$ at $37^{\circ} \mathrm{C}$ in a $5 \% \mathrm{CO} 2 / 95 \%$ air atmosphere. The cells were planted in a 6-well plate and treated with DMEM, AuNP or U2-AuNP, respectively. After $24 \mathrm{~h}$, cells were lysed in RIPA buffer supplemented with protease inhibitors. After protein quantification with a BCA protein assay kit (Thermo Scientific), $20 \mu \mathrm{g}$ protein was detected by $8 \%$ SDS-PAGE gels and blotted onto polyvinylidene fluoride (PVDF) membranes (Millipore, Bedford, MA). The membranes were incubated with the following specific antibodies: anti-pEGFR (tyr1038), anti-EGFR (Santa Cruz Biotechnology, Santa Cruz, CA), anti-pH2AX (S139), anti-pChk2 (T68), anti-Chk2 (Cell Signaling Technology, Danvers, MA), anti-pATM (Ser1981), antiATM (Cell Signaling Technology, Danvers, MA), anti53BP1 (Cell Signaling Technology, Danvers, MA) and anti- $\beta$-actin (Boster Biological Technology, China). A secondary HRP-conjugated antibody was used for immunodetection, and an ECL Western blotting kit (Bio-Rad) was used to visualize the protein bands. Finally, the results were analyzed with ImageJ software.

\section{Stereotactic Intracranial Implantation of Tumor Cells}

All animal experiments were conducted under the approval of the Animal Care and Use Committee of Southern Medical University. In addition, the animal experiments were performed in accordance with the "Administrative Measures for Experimental Animals of Southern Medical University" and "Guidelines for Ethical Review of Animal Experiments of Southern Medical University". Male BALB/C nude mice were 4-6 weeks old and kept in a specific pathogen-free environment. To establish a U87-EGFRvIII animal model, $5 \times 10^{5}$ U87-EGFRvIII cells in $25 \mu \mathrm{L}$ were resuspended after transduction with an eGFP lentivirus for $48 \mathrm{hr}$. The cells were injected into $\mathrm{BALB} / \mathrm{c}$ nude mice, and tumors were grown for 10 days and then randomized into two groups: blank group $(n=6)$ and U2-AuNP group $(n=6)$. Mice were anesthetized with pentobarbital sodium $(10 \mathrm{~mL} / \mathrm{kg})$ and placed face down on a stereotactic device. A burr hole was made $(-2.0 \mathrm{~mm}, 1.25 \mathrm{~mm}, 3.0 \mathrm{~mm})$ in the striatum area using a dental microdrill, with the anterior fontanelle as the origin. Then, $5 \mu \mathrm{L}$ cell suspension containing $1 \times 10^{5}$ U87-EGFRvIII cells was injected over $10 \mathrm{~min}$. The needle was then retracted slowly after a period of $10 \mathrm{~min}$ total time left in the brain. The burr hole was closed with bone wax, the scalp was sutured, and the animals were moved to a heated environment for postoperative recovery.

\section{Therapeutic Effect of U2-AuNP on GBM-Bearing Mice}

Ten days after the injection of tumor cells, tumors in the striatum area were confirmed by the IVIS system (Bruker FX Pro imaging system). $\mathrm{NaCl}$ or APC-CY7labeled U2-AuNP was injected through the tail vein in a volume of $0.1 \mathrm{~mL}$. Mice were sacrificed after living imaging, and the brains of mice were harvested to create frozen sections and photographed by using a laser scanning confocal microscope. Images were processed and analyzed with Adobe Photoshop and Illustrator.

\section{Statistical Analysis}

All data are expressed as mean \pm SD. One-way ANOVA or Student's $t$-test was used for multiple-group or two-group analysis, respectively. A value of $\mathrm{p}<0.05$ was considered statistically significant. All experiments were performed at least 3 times. 


\section{Results}

\section{Characteristics of the Aptamer-Gold Nanoparticle Compounds}

$\mathrm{U} 2$ is a DNA aptamer obtained by Cell-SELEX technology using U87MG, U87EGFR and U87-EGFRvIII cells; it has been verified that $\mathrm{U} 2$ inhibits the proliferation and invasion of U87-EGFRvIII cell lines by inhibiting the EGFR-related signaling pathway. To improve the concentration of aptamer U2 in the brain tumor environment, we modified the aptamer with a thiol group that conjugates to the AuNPs surface through an Au-S bond; the schematic representation of the process of aptamer U2-AuNP conjugation is shown in Figure 1A. It is well known that the gold nanoparticle solution exhibits a characteristic absorption peak at $520 \mathrm{~nm}$; the absorption peak of AuNPs is shifted to $522 \mathrm{~nm}$ when aptamers are conjugated to AuNPs (Figure 1B). Further experiments showed that aptamers connect evenly around the gold nanoparticle, while the zeta potential of U2-AuNP $(-12.3 \mathrm{mV})$ showed little change compared to that of naked AuNPs (-11.4mV) (Figure 1C). Besides, the size of U2AuNP was closed to $60.23 \mathrm{~nm}$ while AuNP was closed to
$19.44 \mathrm{~nm}$ (data not shown), consistent with the TEM results. As shown in Figure 1D, naked AuNPs are denatured and deposited at the sample site, but U2-AuNP, aptamer-AuNP or aptamers $\mathrm{U} 2$ were driven as well as separated in the agarose gel electrophoresis.

\section{Effects of U2-AuNP on U87-EGFRvIll Cell Lines in vitro}

GBM is characterized by excessive proliferation and strong invasion. To investigate the inhibitory effects of U2-AuNP on GBM cell lines, we performed proliferation and invasion experiments. First, we tested whether the U2-AuNP complex could inhibit the proliferation of U87-EGFRvIII cell lines by using the Cell Light EdU DNA Imaging Kit.

EdU (5-ethynyl-2'-deoxyuridine) was effectively detected with a fluorochrome-conjugated azide when EdU was incorporated into cellular DNA during tumor cell proliferation. The proliferation ability of GBM cells was assessed by counting EdU fluorescent positive cells after standardization. Hence, the benchmark was the EdU-positive rate of U87-EGFRvIII cell
A

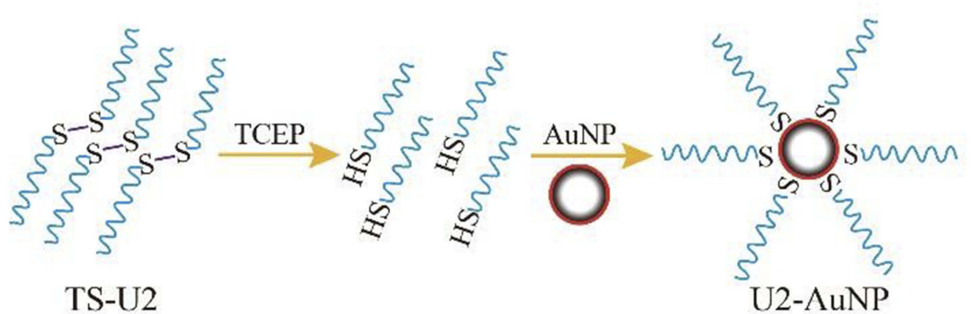

C
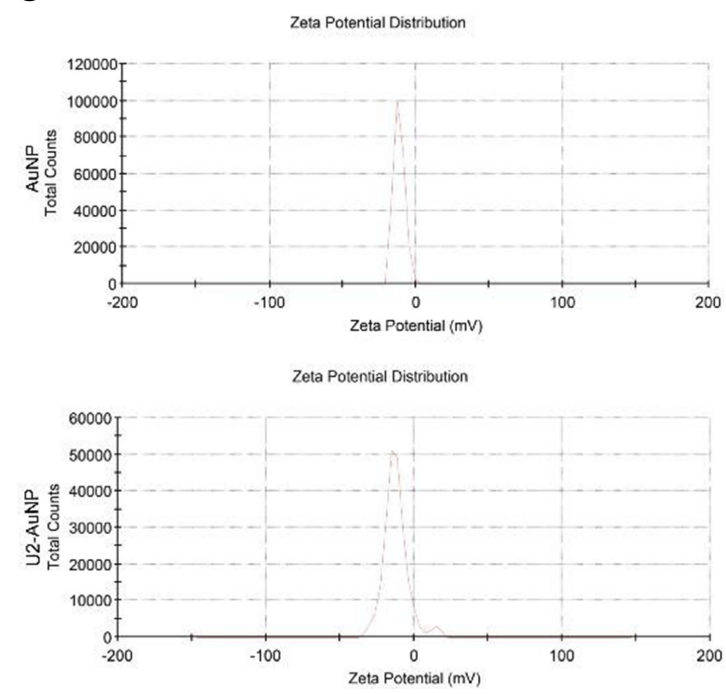

B

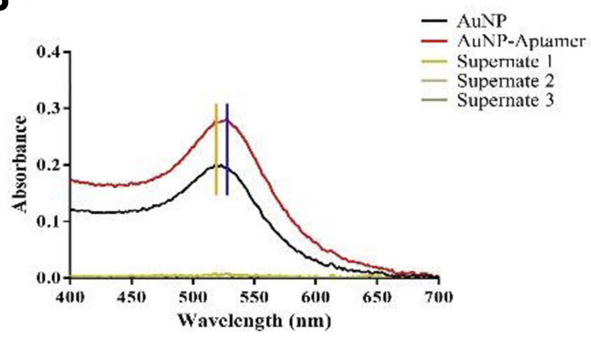

$\mathbf{D}$
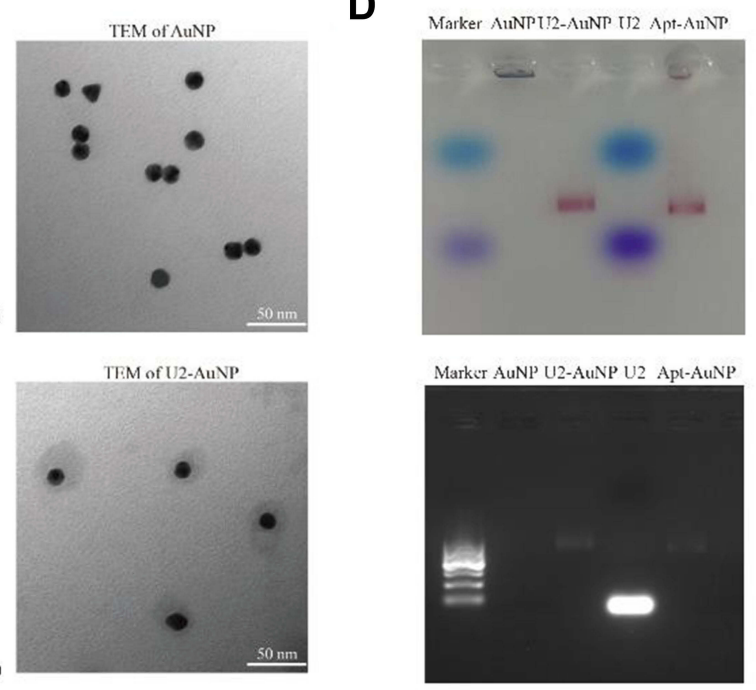

Figure I Characterization of U2-AuNP. (A) Schematic view of the aptamer conjugation with gold nanoparticle. (B) Absorption spectra and absorption peak of aptamerAuNP. (C) DLS and TEM images of U2-AuNP complex. (D) Agarose gel electrophoresis of U2-AuNP complex. 
lines treated with DMEM. As shown in Figure 2A, EdUpositive cells obviously decreased after treatment with U2AuNP. Compared to the number of cells treated with AuNPs (100 $\mathrm{nM}$ or $150 \mathrm{nM}$ ), the percentage of U87-EGFRvIII cells treated with U2-AuNP was reduced from $30 \%$ to $15 \%$ (Figure 2B). In addition, we observed that the proliferation rate decreased with increasing concentrations of U2-AuNP.

Next, we assessed the effect of U2-AuNP on the invasion capacity of GBM cells by using a Transwell chamber coated with a Matrigel membrane. Using this assay, we found that the invasion rate of U87-EGFRvIII cells after U2-AuNP treatment for $24 \mathrm{hr}$ was significantly reduced compared with the invasion rate of cells after DMEM or AuNP treatment (Figure 2C and D). These results indicated that U2-AuNP inhibits the proliferation and invasion capacity of U87-EGFRvIII cells.

\section{Inhibition Mechanism of U2-AuNP to U87-EGFRvIll Cells}

We analyzed the mechanism of U2-AuNP inhibition on the proliferation and invasion of U87-EGFRvIII cells. In our previous work, we found that aptamer $\mathrm{U} 2$ inhibits the proliferation of U87-EGFRvIII cells by inhibiting the autophosphorylation activity of EGFRvIII and its downstream signaling pathway. After U2-AuNP treatment, we collected the cell lysates and immunoblotted them with the respective antibodies. Western blotting results showed that the phosphorylation level of EGFRvIII decreased significantly after U2-AuNP treatment, while total EGFRvIII showed no obvious change, which explained why U2-AuNP inhibits the proliferation and invasion of U87-EGFRvIII cells (Figure 3A). Based on previous reports, gold nanomaterials affect some signaling pathways in DNA damage repair. Herein, we detected the expression of 53BP1 and the phosphorylation of ATM (ataxia telangiectasia mutated), which are critical during the response to DNA damage. As indicated in Figure 3B, D, and E, the phosphorylation level of ATM, the expression of the key protein 53BP1 (binding protein 1), and the phosphorylation level of downstream Chk2, decreased significantly after $24 \mathrm{hr}$ treatment of U2-AuNP compared to the levels in the other two control groups. However, the phosphorylation level of H2A.X showed no change after treatment with U2-AuNP $24 \mathrm{hr}$ (Figure 3C).

\section{U2-AuNP Prolonged the Survival Time of GBM-Bearing Mice}

To identify the brain-targeting effect of U2-AuNP, an intracranial GBM mouse model was prepared by injecting U87-
EGFRvIII cells expressing eGFP by intrastriatal injection by using a stereotaxic method. After 10 days of cell implantation, APC-CY7-labeled U2-AuNP were intravenously injected into the mice via the tail vein. Twenty-four hours later, the mice were sacrificed, and the brains of the mice were harvested for frozen sectioning and photographed by a laser scanning confocal microscope. As shown in Figure 4A, red fluorescence was detected in GBM-brain slices after injection of APC-CY7 -labeled U2-AuNP, while no fluorescence signal was found in the APC-CY7 group (Figure 4B). This result suggested that U2-AuNP could efficiently cross the BBB and enter the tumor region.

Furthermore, we wanted to determine whether U2AuNP therapy might inhibit the progression of GBM in tumor-bearing mice. After 10 days of tumor cell implantation, $\mathrm{U} 2-\mathrm{AuNP}$ or $\mathrm{NaCl}$ in the same volume were injected through the tail vein into the tumor-bearing mice once every 3 days. The results also showed that the mice treated with U2-AuNP had a prolonged survival time compared with that of those treated with $\mathrm{NaCl}$ (Figure 4C). In addition, the mean survival time of mice treated with U2-AuNP was 30 days, which was longer than that of $\mathrm{NaCl}$-treated mice (24 days) (Figure 4D).

\section{Discussion}

GBM represents one of the most frequent and aggressive brain tumors and is associated with a relatively higher proportion of cancer-related deaths. ${ }^{21}$ It has been reported that EGFR is one of the most frequent effectors of adult GBM, ${ }^{6}$ and GBM is known to have a deletion in the EGFR extracellular domain to form EGFRvIII or amplification and coexpression of the wild-type EGFR allele, ${ }^{22}$ which indicates EGFRvIII is an appropriate target for GBM therapy. In recent years, EGFRvIII-directed CAR T cells ${ }^{23}$ and anti-EGFRvIII vaccine rindopepimut have shown unsatisfactory therapeutic effects in trials. ${ }^{24}$ The reasons why GBM-targeted therapies only have moderate effects are mainly related to limits of brain penetration, redundant activating pathways and intratumoral heterogeneity. ${ }^{25}$ It recently became clear that gold nanoparticles, as one of the most widely used high capacity nanomaterials, show a radiosensitization capacity because AuNPs can absorb considerably more photons to increase the local dose of radiation in cells and then damage DNA directly or indirectly inducing cell apoptosis. ${ }^{26}$ Moreover, AuNPs between 10 and $20 \mathrm{~nm}$ can cross the blood-brain barrier after covalently binding with miRNA. ${ }^{19,27}$ Therefore, we designed the U2-AuNP complex to help the 
A

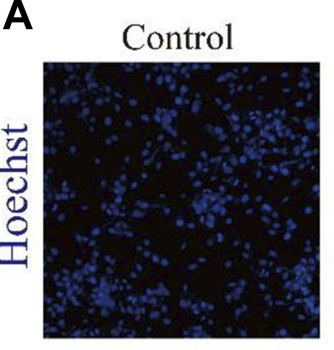

AuNP-100nM
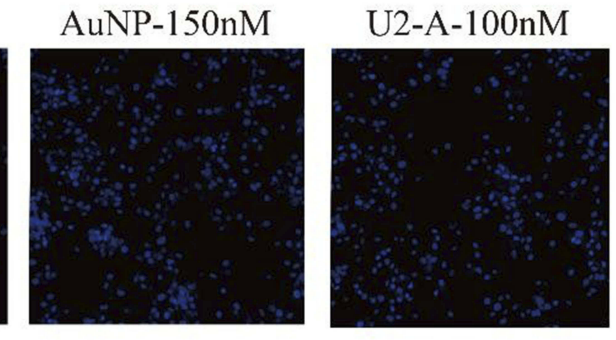

U2-A-150nM
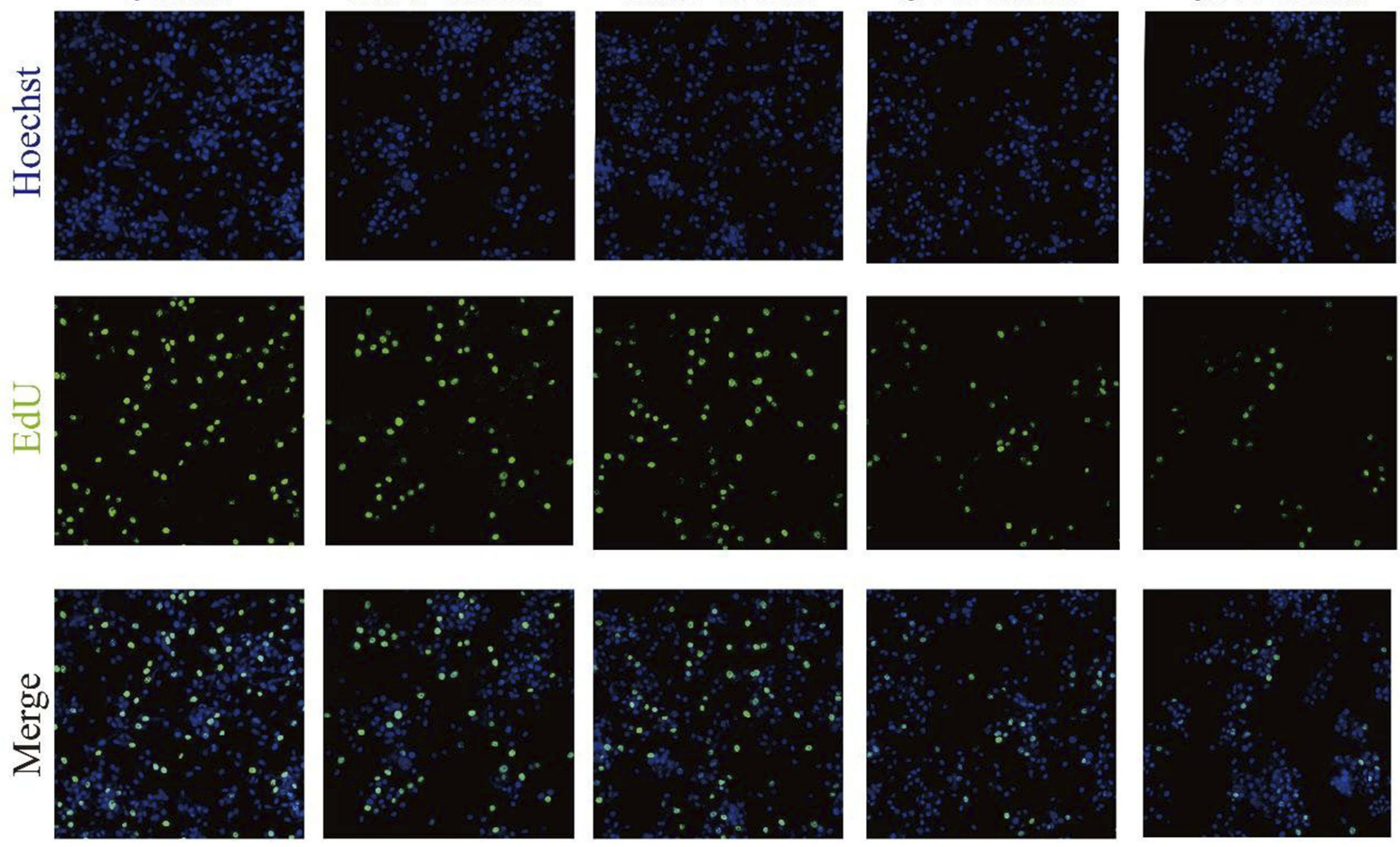

B

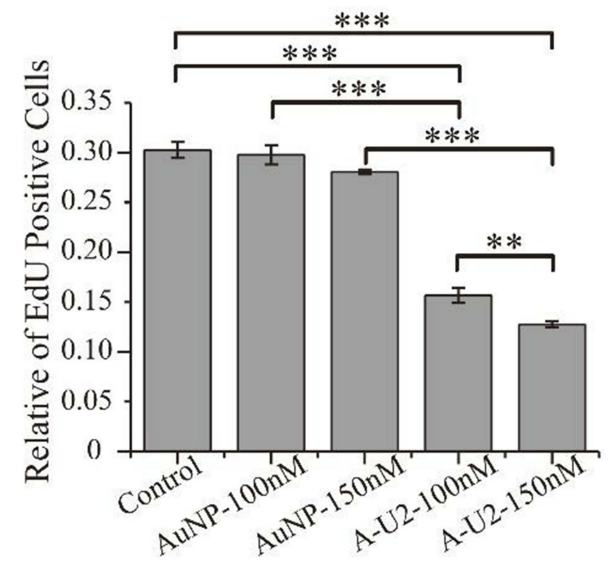

D

Control

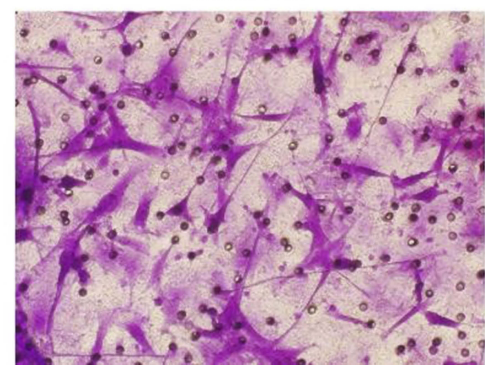

C

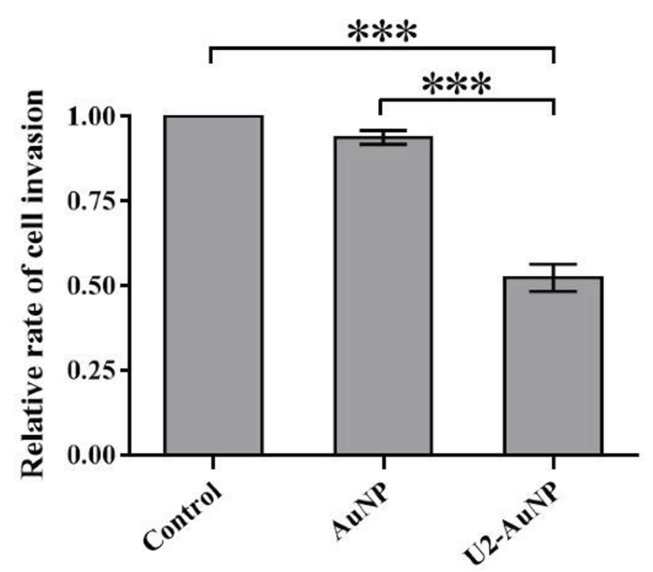

AuNP

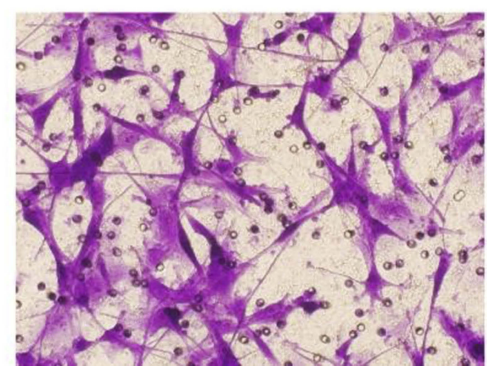

U2-AuNP

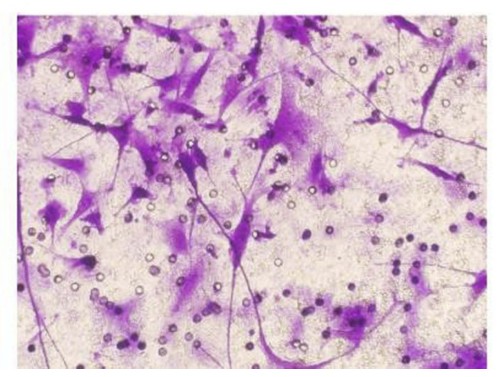

Figure 2 U2-AuNP affects U87-EGFRvill cell proliferation and invasion. (A) U2-AuNP (U2-A) reduces the rate of EdU-positive cells. (B) Data of EdU assay are presented as the percentage of EdU-positive cells in the presence of U2-AuNP(A-U2) compared with AuNPs and control group. (C) Data of invasion assay are presented as the percentage of invaded cells in the presence of U2-AuNP compared with AuNPs and control group. (D) Invasion of U87-EGFRvlll cells through matrigel was evaluated in the presence of U2-AuNP or AuNPs for the indicated times. $* * * P<0.001$; $* * P<0.01$. 

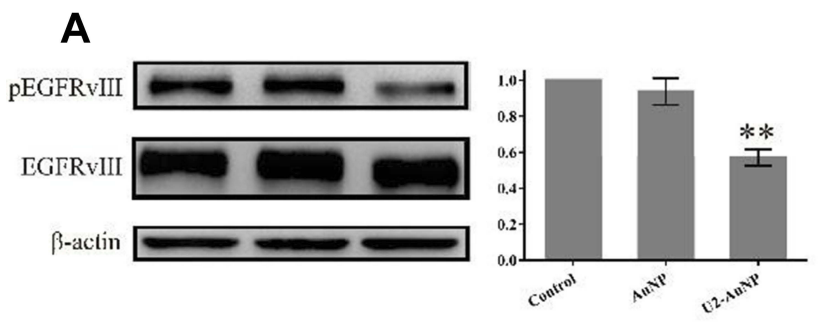

B
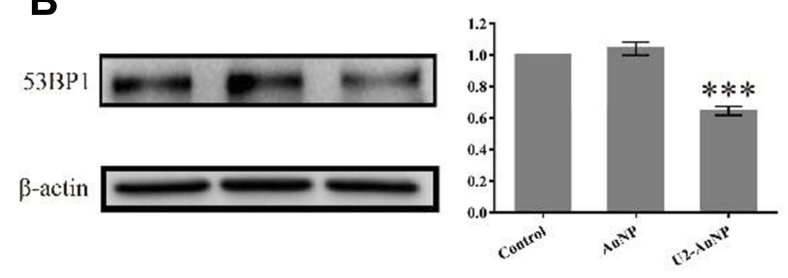

D
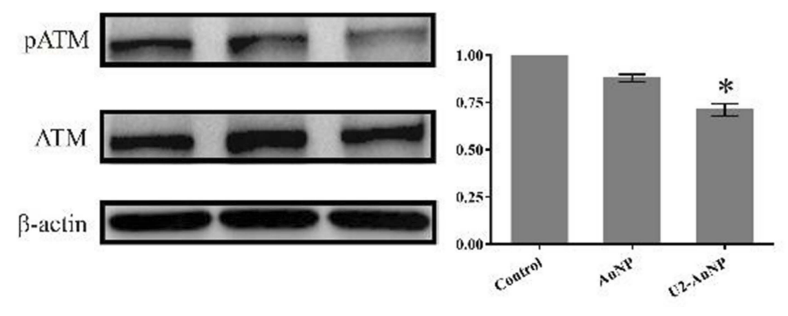

C
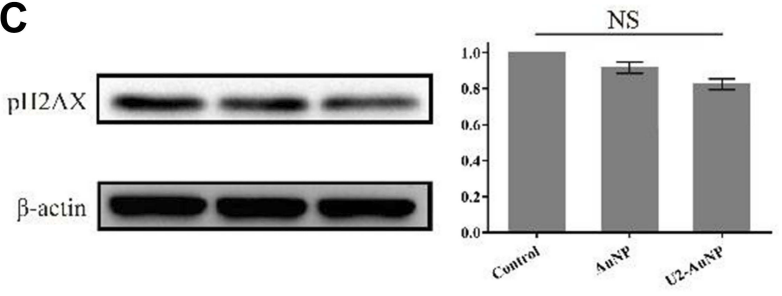

E

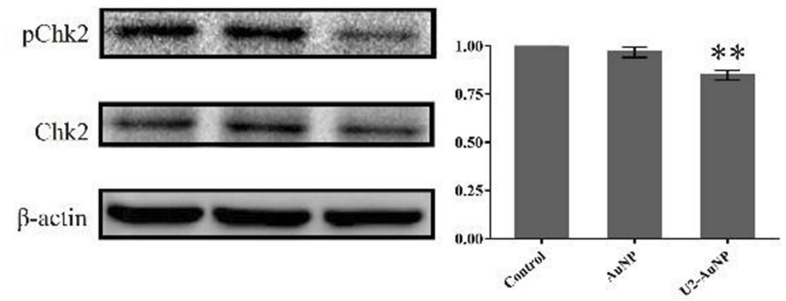

Figure 3 U2-AuNP inhibits the activation of EGFRvIll and DNA injury repair pathway. (A) Western Blot analysis of the phosphorylation level of EGFRvill. Setting the values of the relative ratio of untreated cells to $100 \%$, the values below the blot indicate the ratio of PEGFR to total EGFR signal levels after normalization with the $\beta$-actin signal level. (B-E) Immunoblotted for phosphorylated and total markers related to DNA damage repair, as indicated. $* * * P<0.001 ; * * P<0.01 ; * P<0.05 ; N S:$ no significance.

EGFRvIII-targeted aptamer U2 cross the blood-brain barrier and reach the tumor to inhibit GBM.

In our study, we investigated the characteristics of U2AuNP and the effects of U2-AuNP on U87-EGFRvIII cells at the cellular level and in an animal model. By using agarose gel electrophoresis and transmission electron microscopy, we found that aptamer U2 modification with a thiol group can create stably binding to AuNPs through the Au-S bond. Next, we focused on the effects of the U2-AuNP complex in U87-EGFRvIII cells. According to previous reports, inhibiting the EGFR-related pathway can suppress the proliferation and invasion of tumors. ${ }^{28-31}$ Consistent with previous studies, we observed that the proliferation and invasion that cause GBM aggressive growth and difficult complete resection of the cancer were reduced significantly after $24 \mathrm{hr}$ treatment with U2-AuNP. Gold nanoparticles with aptamer have been widely applied in biosensors and imaging but not in brain-targeting delivery carriers. ${ }^{32-34}$ However, no study has been performed to evaluate the effects of aptamers conjugated with gold nanoparticles to treat glioma. In this report, we tentatively propose that the U2-AuNP complex could effectively cross the blood-brain barrier and accumulate around GBM in vivo. In addition to the ability to cross the blood-brain barrier, we identified that U2-AuNP treatment by intravenous injections can prolong the survival time of GBM-bearing mice.

It is widely accepted that the inhibition of EGFR suppresses tumor cell proliferation and invasion in GBM. ${ }^{35-37}$ Consistent with these findings, our manuscript also demonstrates that $24 \mathrm{hr}$ treatment with U2-AuNP could significantly reduce the phosphorylation level of EGFRvIII. To better understand the mechanism of U2-AuNP in GBM, we detected DNA damage and repair-related pathways. Interestingly, treatment with U2-AuNP can decrease the expression level of 53BP1 (binding protein 1) and the phosphorylation level of ATM and Chk2, which are critical in the response to DNA damage. ${ }^{38-40}$ In recent years, exposure to gold nanoparticles has been reported to cause transient DNA damage due to the generation of alkali-labile sites and oxidative stress with a decrease in glutathione. ${ }^{41,42}$ However, the phosphorylation level of H2A.X showed no change after treatment with U2-AuNP $24 \mathrm{hr}$. We considered that this phenomenon might be caused by a long U2-AuNP treatment time since DNA double-strand breaks (DSBs) happen within several hours. 
A
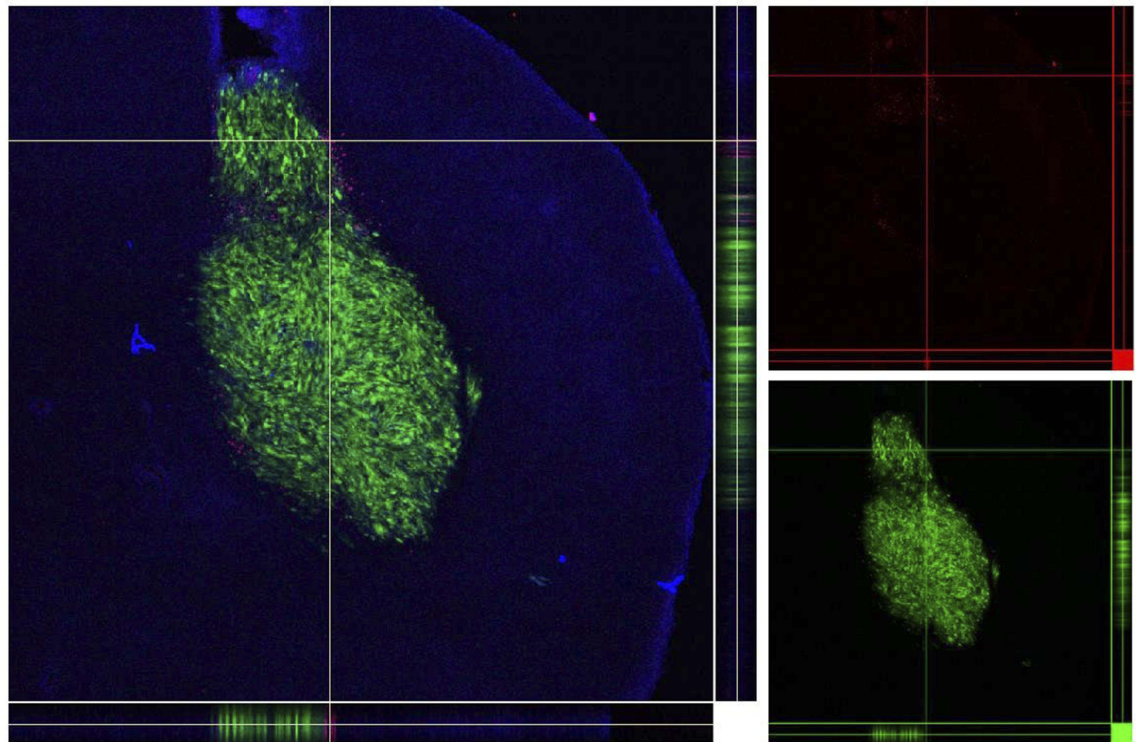

B
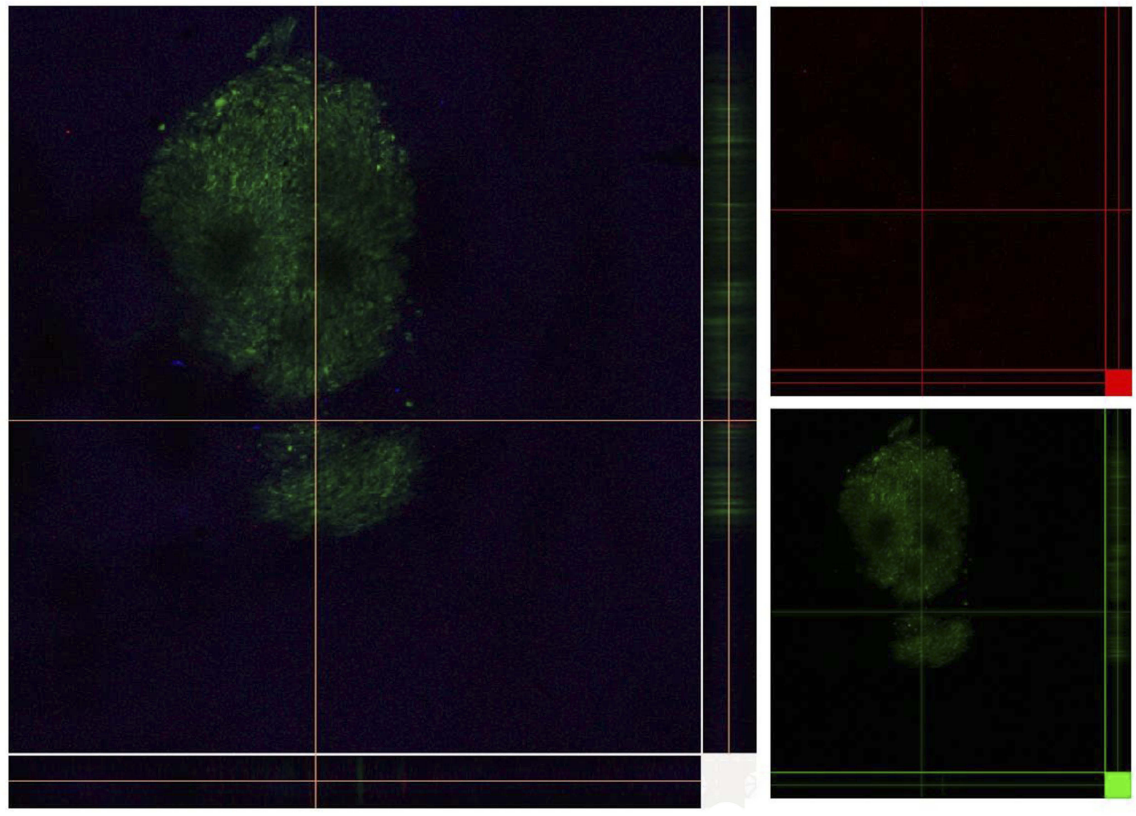

C

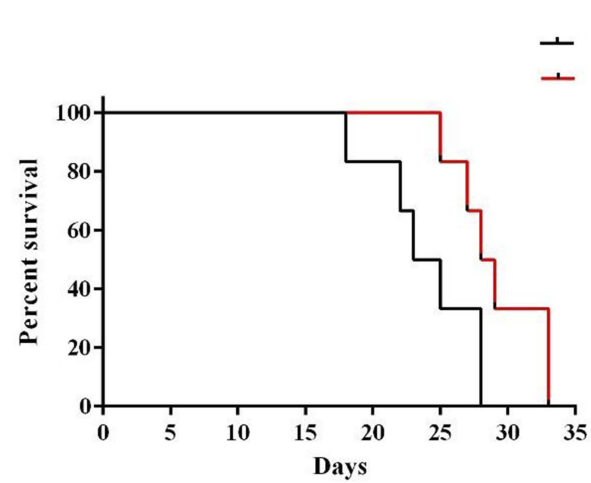

D

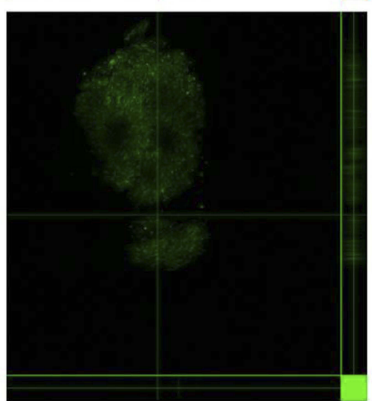

$-\mathrm{NaCl}$

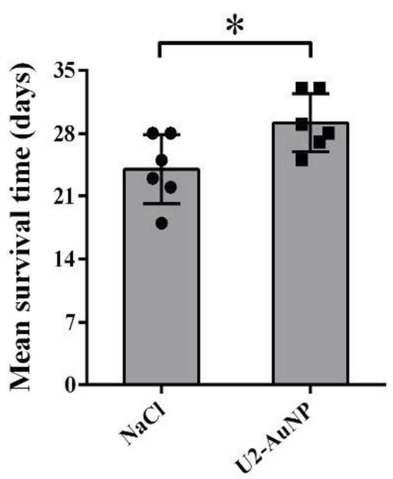

Figure 4 U2-AuNP affects the survival of animal model. (A) Z stack of GBM tumor after injection with Cy7-labeled U2-AuNP for $24 \mathrm{~h}$. (B) Z stack of GBM tumor after injection with Cy7 solution for 24 h. Red: Cy7 labeled; Green: U87-EGFRvill cells. (C) Survival curve and (D) mean survival time of GBM-bearing mice treated with the U2AuNP or $\mathrm{NaCl}$. $* P<0.05$. 
When DNA damage occurs within $6 \mathrm{hr}$, a change in the phosphorylation level of H2A.X can be detected. ${ }^{9,43}$

\section{Conclusion}

U2-AuNP shows outstanding inhibition of GBM in vitro and in vivo. Based on our previous research, we found that the U2-AuNP complex exhibits potential value as a multifunctional therapeutic strategy for inhibiting the EGFR-related pathway and preventing DNA damage repair to GBM. Further studies will focus on further research on the molecular mechanism involving AuNP-based aptamers and DNA damage repair pathways.

\section{Acknowledgments}

This project was supported by the National Natural Science Foundation of China (No. 81871418, 81771484), the Natural Science Foundation of Guangdong Province, China (No. 2018A030313835), the Undergraduate Innovation and Entrepreneurship Training Program of Southern Medical University (No. 201912121234), the Medical Scientific Research Foundation of Guangdong Province (B2019185), the Scientific Research Startup Program of Southern Medical University (PY2018N084), the Program for Changjiang Scholars and Innovative Research Team in University (No. IRT_16R37), and the Guangzhou Science and Technology Project (No.201707020027).

\section{Disclosure}

The authors report no conflicts of interest in this work.

\section{References}

1. Varlet P, Le Teuff G, Le Deley MC, et al. WHO grade has no prognostic value in the pediatric high-grade glioma included in the HERBY trial. Neuro-Oncology. 2020;22(1):116-127. doi:10.1093/neuonc/noz142

2. Lozada-Delgado EL, Grafals-Ruiz N, Vivas-Mejia PE. RNA interference for glioblastoma therapy: innovation ladder from the bench to clinical trials. Life Sci. 2017;188:26-36. doi:10.1016/j.lfs.2017.08.027

3. Roy LO, Poirier MB, Fortin D. differential expression and clinical significance of transforming growth factor-beta isoforms in GBM tumors. Int J Mol Sci. 2018;19(4):1113. doi:10.3390/ijms19041113

4. Molinaro AM, Taylor JW, Wiencke JK, Wrensch MR. Genetic and molecular epidemiology of adult diffuse glioma. Nat Rev Neurol. 2019;15(7):405-417. doi:10.1038/s41582-019-0220-2

5. Chen P, Zhao D, Li J, et al. Symbiotic macrophage-glioma cell interactions reveal synthetic lethality in PTEN-null glioma. Cancer Cell. 2019;35(6):868-884 e866. doi:10.1016/j.ccell.2019.05.003

6. Jones DTW, Banito A, Grunewald TGP, et al. Molecular characteristics and therapeutic vulnerabilities across paediatric solid tumours. Nat Rev Cancer. 2019;19(8):420-438. doi:10.1038/s41568-019-0169-x

7. Jutten B, Keulers TG, Peeters HJM, et al. EGFRvIII expression triggers a metabolic dependency and therapeutic vulnerability sensitive to autophagy inhibition. Autophagy. 2018;14(2):283-295. doi:10.1080/ 15548627.2017.1409926
8. Lammering G, Hewit TH, Holmes M, et al. Inhibition of the type III epidermal growth factor receptor variant mutant receptor by dominant-negative EGFR-CD533 enhances malignant glioma cell radiosensitivity. Clin Cancer Res. 2004;10(19):6732-6743. doi:10.1158/ 1078-0432.CCR-04-0393

9. Zhang X, Peng L, Liang Z, et al. Effects of aptamer to U87-EGFRvIII cells on the proliferation, radiosensitivity, and radiotherapy of glioblastoma cells. Mol Ther Nucleic Acids. 2018;10:438-449. doi:10.1016/j. omtn.2018.01.001

10. Lao YH, Phua KK, Leong KW. Aptamer nanomedicine for cancer therapeutics: barriers and potential for translation. ACS Nano. 2015;9 (3):2235-2254. doi:10.1021/nn507494p

11. Zhu G, Chen X. Aptamer-based targeted therapy. Adv Drug Deliv Rev. 2018;134:65-78. doi:10.1016/j.addr.2018.08.005

12. Tan Y, Shi YS, Wu XD, et al. DNA aptamers that target human glioblastoma multiforme cells overexpressing epidermal growth factor receptor variant III in vitro. Acta Pharmacol Sin. 2013;34 (12):1491-1498. doi:10.1038/aps.2013.137

13. Aparicio-Blanco J, Martin-Sabroso C, Torres-Suarez AI. In vitro screening of nanomedicines through the blood brain barrier: a critical review. Biomaterials. 2016;103:229-255. doi:10.1016/j. biomaterials.2016.06.051

14. Saraiva C, Praca C, Ferreira R, Santos T, Ferreira L, Bernardino L. Nanoparticle-mediated brain drug delivery: overcoming blood-brain barrier to treat neurodegenerative diseases. $J$ Control Release. 2016;235:34-47. doi:10.1016/j.jconrel.2016.05.044

15. Sweeney MD, Sagare AP, Zlokovic BV. Blood-brain barrier breakdown in Alzheimer disease and other neurodegenerative disorders. Nat Rev Neurol. 2018;14(3):133-150. doi:10.1038/nrneurol.2017. 188

16. Pollak TA, Drndarski S, Stone JM, David AS, McGuire P, Abbott NJ. The blood-brain barrier in psychosis. Lancet Psychiatry. 2018;5 (1):79-92. doi:10.1016/S2215-0366(17)30293-6

17. Her S, Jaffray DA, Allen C. Gold nanoparticles for applications in cancer radiotherapy: mechanisms and recent advancements. $A d v$ Drug Deliv Rev. 2017;109:84-101. doi:10.1016/j.addr.2015.12.012

18. Liu J, Peng Q. Protein-gold nanoparticle interactions and their possible impact on biomedical applications. Acta Biomater. 2017;55:13-27. doi:10.1016/j.actbio.2017.03.055

19. Kouri FM, Hurley LA, Daniel WL, et al. miR-182 integrates apoptosis, growth, and differentiation programs in glioblastoma. Genes Dev. 2015;29(7):732-745. doi:10.1101/gad.257394.114

20. Wu X, Liang H, Tan Y, et al. Cell-SELEX aptamer for highly specific radionuclide molecular imaging of glioblastoma in vivo. PLoS One. 2014;9(6):e90752. doi:10.1371/journal.pone.0090752

21. Lawrie TA, Gillespie D, Dowswell T, et al. Long-term neurocognitive and other side effects of radiotherapy, with or without chemotherapy, for glioma. Cochrane Database Syst Rev. 2019;8:CD013047.

22. Sturm D, Bender S, Jones DT, et al. Paediatric and adult glioblastoma: multiform (epi)genomic culprits emerge. Nat Rev Cancer. 2014;14(2):92-107. doi:10.1038/nrc3655

23. O'Rourke DM, Nasrallah MP, Desai A, et al. A single dose of peripherally infused EGFRvIII-directed CAR T cells mediates antigen loss and induces adaptive resistance in patients with recurrent glioblastoma. Sci Transl Med. 2017;9(399):eaaa0984. doi:10.1126/ scitranslmed.aaa0984

24. Malkki H. Trial watch: glioblastoma vaccine therapy disappointment in Phase III trial. Nat Rev Neurol. 2016;12(4):190. doi:10.1038/ nrneurol.2016.38

25. Chen R, Smith-Cohn M, Cohen AL, Colman H. Glioma subclassifications and their clinical significance. Neurotherapeutics. 2017;14 (2):284-297. doi:10.1007/s13311-017-0519-x

26. Chugh H, Sood D, Chandra I, Tomar V, Dhawan G, Chandra R. Role of gold and silver nanoparticles in cancer nano-medicine. Artif Cells Blood Substit Biotechnol. 2018;46(sup1):1210-1220. doi:10.1080/ 21691401.2018.1449118 
27. Betzer O, Shilo M, Opochinsky R, et al. The effect of nanoparticle size on the ability to cross the blood-brain barrier: an in vivo study. Nanomedicine. 2017;12(13):1533-1546. doi:10.2217/nnm-20170022

28. Hung WY, Chang JH, Cheng Y, et al. Leukocyte cell-derived chemotaxin 2 retards non-small cell lung cancer progression through antagonizing MET and EGFR activities. Cell Physiol Biochem. 2018;51(1):337-355. doi:10.1159/000495233

29. Momeny M, Esmaeili F, Hamzehlou S, et al. The ERBB receptor inhibitor dacomitinib suppresses proliferation and invasion of pancreatic ductal adenocarcinoma cells. Cell Oncol. 2019;42(4):491-504. doi:10.1007/s13402-019-00448-w

30. Zeng W, Zhu JF, Liu JY, et al. miR-133b inhibits cell proliferation, migration and invasion of esophageal squamous cell carcinoma by targeting EGFR. Biomed Pharmacother. 2019;111:476-484. doi:10. 1016/j.biopha.2018.12.057

31. Zhang Y, Wei Y, Li X, et al. microRNA-874 suppresses tumor proliferation and metastasis in hepatocellular carcinoma by targeting the DOR/EGFR/ERK pathway. Cell Death Dis. 2018;9(2):130. doi:10.1038/s41419-017-0131-3

32. Haghighi FH, Binaymotlagh R, Mirahmadi-Zare SZ, Hadadzadeh H. Aptamer/magnetic nanoparticles decorated with fluorescent gold nanoclusters for selective detection and collection of human promyelocytic leukemia (HL-60) cells from a mixture. Nanotechnology. 2020;31(2):025605. doi:10.1088/1361-6528/ab484a

33. Shayesteh $\mathrm{OH}$, Ghavami R. A novel label-free colorimetric aptasensor for sensitive determination of PSA biomarker using gold nanoparticles and a cationic polymer in human serum. Spectrochim Acta a Mol Biomol Spectrosc. 2020;226:117644. doi:10.1016/j.saa.2019. 117644

34. Wang Y, Wang B, Xiong X, Deng S. Gold nanoparticle-based signal enhancement of an aptasensor for ractopamine using liquid crystal based optical imaging. Mikrochim Acta. 2019;186(11):697. doi:10. 1007/s00604-019-3811-0
35. Binder ZA, Thorne AH, Bakas S, et al. Epidermal growth factor receptor extracellular domain mutations in glioblastoma present opportunities for clinical imaging and therapeutic development. Cancer Cell. 2018;34 (1):163-177 e167. doi:10.1016/j.ccell.2018.06.006

36. Cheng F, Guo D. MET in glioma: signaling pathways and targeted therapies. J Exp Clin Cancer Res. 2019;38(1):270. doi:10.1186/ s13046-019-1269-x

37. Sun Z, Xue H, Wei Y, et al. Mucin O-glycosylating enzyme GALNT2 facilitates the malignant character of glioma by activating the EGFR/ PI3K/Akt/mTOR axis. Clin Sci. 2019;133(10):1167-1184. doi:10. $1042 / \mathrm{CS} 20190145$

38. Bartova E, Legartova S, Dundr M, Suchankova J. A role of the 53BP1 protein in genome protection: structural and functional characteristics of 53BP1-dependent DNA repair. Aging. 2019;11(8):2488-2511. doi:10. 18632/aging.v11i8

39. Campillo-Marcos I, Lazo PA. Olaparib and ionizing radiation trigger a cooperative DNA-damage repair response that is impaired by depletion of the VRK1 chromatin kinase. J Exp Clin Cancer Res. 2019;38(1):203. doi:10.1186/s13046-019-1204-1

40. Miyake T, Shimada M, Matsumoto Y, Okino A. DNA damage response after ionizing radiation exposure in skin keratinocytes derived from human-induced pluripotent stem cells. Int J Radiat Oncol Biol Phys. 2019;105(1):193-205. doi:10.1016/j.ijrobp.2019.05.006

41. May S, Hirsch C, Rippl A, et al. Transient DNA damage following exposure to gold nanoparticles. Nanoscale. 2018;10(33):15723-15735. doi:10.1039/C8NR03612H

42. Huang C, Bao Q, Hunting D, Zheng Y, Sanche L. Conformationdependent DNA damage induced by gold nanoparticles. J Biomed Nanotechnol. 2013;9(5):856-862. doi:10.1166/jbn.2013.1578

43. West KL, Kelliher JL, Xu Z, et al. LC8/DYNLL1 is a 53BP1 effector and regulates checkpoint activation. Nucleic Acids Res. 2019;47 (12):6236-6249. doi:10.1093/nar/gkz263
International Journal of Nanomedicine

\section{Publish your work in this journal}

The International Journal of Nanomedicine is an international, peerreviewed journal focusing on the application of nanotechnology in diagnostics, therapeutics, and drug delivery systems throughout the biomedical field. This journal is indexed on PubMed Central, MedLine, CAS, SciSearch ${ }^{\mathbb{}}$, Current Contents ${ }^{\mathbb{R}} /$ Clinical Medicine,
Journal Citation Reports/Science Edition, EMBase, Scopus and the Elsevier Bibliographic databases. The manuscript management system is completely online and includes a very quick and fair peer-review system, which is all easy to use. Visit http://www.dovepress.com/ testimonials.php to read real quotes from published authors. 